\title{
Tecnologia Assistiva vibrotátil para a educação musical de surdos
}

\author{
Vibrotactile Assistive Technology for the musical education of the deaf \\ Tecnología de Asistencia vibrotáctil para la educación musical para personas sordas
}

\author{
André Leite de Farias \\ ORCID: https://orcid.org/0000-0001-6096-4619 \\ Universidade Católica de Brasília, Brasil \\ E-mail: andreleitedefarias@gmail.com \\ Ana Keully Gadelha dos Santos Darub \\ ORCID: https://orcid.org/0000-0001-7165-5563 \\ Universidade Federal do Acre, Brasil \\ E-mail: ana.keully@hotmail.com \\ Pricila Kohls dos Santos \\ ORCID: https://orcid.org/0000-0002-3349-4057 \\ Universidade Católica de Brasília, Brasil \\ E-mail: pricila.kohls@gmail.com
}

\begin{abstract}
Resumo
O objetivo deste artigo foi analisar as possibilidades e as limitações da Tecnologia Assistiva vibrotátil para a educação musical de surdos. Adotamos os pressupostos da pesquisa quantitativa, por meio da abordagem bibliométrica (ÂnguloCuentas et al., 2018), bem como os da pesquisa qualitativa, por meio da Análise Textual Discursiva (ATD) proposta por Moraes e Galiazzi (2006). O escopo da pesquisa bibliométrica compreendeu 721 artigos científicos indexados à plataforma PubMed. Os resultados apontaram que os artigos científicos que pesquisam a interseção música e surdez tratam, principalmente, acerca de implantes cocleares, da percepção de audição, de tom e de fala de seres humanos de ambos os sexos biológicos e de idades variadas, no entanto, notamos a escassez das pesquisas sobre a utilização de Tecnologia Assistiva vibrotátil nessa área. Assim, após inserção dos descritores tactile ou vibrotactile, analisamos, por meio da ATD, 7 artigos indexados à plataforma Pubmed e 5 artigos indexados à Web of Science. Concluímos que, apesar de incipientes, a Tecnologia Assistiva vibrotátil pode contribuir para a educação musical de surdos.
\end{abstract}

Palavras-chave: Educação musical; Surdez; Tecnologia Assistiva.

\begin{abstract}
The aim of this paper was to analyze the possibilities and limitations of vibrotactile Assistive Technology for the musical education of the deaf. We adopted the assumptions of quantitative research, through the bibliometric approach (ÂnguloCuentas et al., 2018), as well as those of qualitative research, through the Textual Discursive Analysis (ATD) proposed by Moraes and Galiazzi (2006). The scope of the bibliometric search comprised 721 scientific articles indexed to the PubMed platform. The results showed that the scientific articles that research the intersection of music and deafness deal mainly with cochlear implants, the perception of hearing, tone and speech in human beings of both biological sexes and of varying ages, however, we noted the scarcity of research on the use of vibrotactile Assistive Technology in this area. Thus, after inserting the descriptors tactile or vibrotactile, we analyzed, through ATD, 7 articles indexed to the Pubmed platform and 5 articles indexed to the Web of Science. We conclude that, despite being incipient, the vibrotactile Assistive Technology can contribute to the musical education of the deaf.
\end{abstract}

Keywords: Musical education; Deafness; Assistive Technology.

\section{Resumen}

El objetivo de este artículo fue analizar las posibilidades y limitaciones de la tecnología de asistencia vibrotáctil para la educación musical de los sordos. Adoptamos los supuestos de la investigación cuantitativa, a través del enfoque bibliométrico (Ângulo-Cuentas et al., 2018), así como los de la investigación cualitativa, a través del Análisis Textual Discursivo (ATD) propuesto por Moraes y Galiazzi (2006). El alcance de la búsqueda bibliométrica comprendió 721 artículos científicos indexados a la plataforma PubMed. Los resultados mostraron que los artículos científicos que investigan la intersección de la música y la sordera tratan principalmente de los implantes cocleares, la percepción de la audición, el tono y el habla en seres humanos de ambos sexos biológicos y de distintas edades, sin embargo, notamos la escasez de investigaciones sobre el uso de tecnología de asistencia vibrotáctil en esta área. Así, luego de insertar los descriptores táctil o vibrotáctil, analizamos, a través de ATD, 7 artículos indexados a la plataforma Pubmed y 5 artículos indexados a la Web of Science. Concluimos que, a pesar de ser incipiente, la Tecnología Asistiva Vibrotáctil puede contribuir a la educación musical de los sordos.

Palabras clave: Educación musical; Sordera; Tecnología de Asistencia. 


\section{Introdução}

Na perspectiva clínica, a surdez pode ser vista como uma condição que afeta negativamente diferentes aspectos da vida social de milhões de pessoas em todo o mundo, é multifatorial, manifesta-se em diferentes intensidades e as atividades culturais centradas na música podem não ser convidativas para surdos (Araújo et al., 2017). De acordo com Borowiec, Hökelmann \& Osiński (2019), isso aumenta o sentimento de exclusão em surdos e afeta negativamente sua autoestima.

Como resposta aos sentimentos de exclusão desencadeados por ambientes educacionais e sociais que se fundamentam exclusivamente na perspectiva clínica, a proposta de educação inclusiva, ao longo dos anos, dedicou-se a questionar práticas sociais e educacionais excludentes e a pensar meios de assegurar o direito de todos à educação. Nessa perspectiva, a surdez deixa de ser vista como uma deficiência e passa a ser compreendida como uma diferença. Por essa razão, os ambientes educacionais e sociais são responsáveis pela realização das adaptações necessárias para a remoção de barreiras que impeçam o usufruto dos direitos educacionais dos cidadãos surdos para que, dessa forma, tenham a condição de sujeitos diferentes respeitados. (Brasil, 2015; Gesser, 2009).

O presente estudo se fundamenta na teoria histórico-cultural de Vigotski sobre a surdez. Dessa forma, compreendemos que a surdez impõe ao sujeito que a possui uma condição diferente na relação entre ele e meio, e que, por vezes, tal condição é considerada como fator limitante para o desenvolvimento deste. Nesse contexto, a deficiência é uma construção social e, por conseguinte, compreendemos que o respeito a essas especificidades devem orientar o planejamento de estratégias de ensino adequadas, pois as diferenças humanas não podem ser tratadas como desvantagem no ambiente educacional, provocando a exclusão de pessoas que não correspondem as expectativas normativas para os aprendizes (Vigotski, 2011; Paula \& Pederiva, 2018; Gesser, 2009; Goffman, 2008).

A partir de tal compreensão, ainda que o sentido da audição não esteja disponível, a educação musical para surdos é possível e as oportunidades de aprendizagem deste campo de conhecimento devem ser as dadas para os alunos surdos, afinal música não é composta exclusivamente de sons, ela é também vibração (Paula \& Pederiva, 2018).

No caso dos alunos surdos, os caminhos sensoriais para esta aprendizagem mudam e o processamento das modalidades restantes passam a ser explorado como estratégia para a aprendizagem (Good, Reed \& Russo, 2014). Para esses autores, tal mudança, conhecida como plasticidade compensatória, resulta em uma experiência sensorial única para os surdos, incluindo a maneira como a música é percebida. Nesse sentido, processos táteis mais cognitivos e complexos, como percepção musical, poderiam ajudar a descobrir capacidades táteis superiores em surdos (Sharp, Bacon \& Champoux, 2020).

A esse respeito, segundo Györgyjakab (2018), os surdos não podem ouvir a música, mas podem vê-la e senti-la, se lhes fornecermos os elementos visuais e vibrotáteis corretos. Tranchant et al. (2017) apontam que a percepção musical vibrotátil é de particular relevância para os surdos, que contam com informações sensoriais não auditivas para sentir a música. É nes se ponto que vislumbramos a importância da utilização da Tecnologia Assistiva como ferramenta para a eliminação de barreiras para a educação musical de surdos.

Assim, tendo em vista as características da estimulação vibrotátil e os dispositivos de Tecnologia Assistiva para a transmissão de informações rítmicas contidas na música que podem ser aplicados à educação musical para surdos, pretendemos investigar as possibilidades e as limitações da Tecnologia Assistiva vibrotátil para a educação musical de surdos.

\section{Metodologia}

O presente artigo adotou os pressupostos da pesquisa quantitativa, por meio da abordagem bibliométrica (ÂnguloCuentas et al., 2018), bem como os da pesquisa qualitativa, por meio da Análise Textual Discursiva (ATD) proposta por Moraes e Galiazzi (2006). 
A abordagem bibliométrica consiste na realização de estudos quantitativos sobre a produção de conhecimentos científicos a respeito de uma determinada área. Nele se discute aspectos como a disseminação e a socialização de estudo sobre o tema da pesquisa (Ribeiro, 2017).

Inicialmente, a fim de apresentar os dados quantitativos bibliográficos, coletados em artigos indexados à plataforma PubMed, utilizamos a análise bibliométrica. Para tal análise, utilizamos, o software Microsoft Excel®, para o cálculo de indicadores de primeiro nível, por contagem de frequências, e o software VOSviewer ${ }^{\circledR}$, para a construção e visualização de redes de coocorrência de termos importantes extraídos de um corpus da literatura científica (Ângulo-Cuentas et al., 2018).

Nesse sentido, utilizamos os descritores: music; deaf or deafness. O escopo dessa pesquisa compreendeu 721 artigos científicos na plataforma PubMed. Após apresentação dos resultados da análise bibliométrica, com o intuito de nos aprofundarmos no tema sobre Tecnologias Assistivas vibrotáteis utilizadas para a educação musical de surdos, discutimos, por meio da ATD, os dados encontrados em 7 artigos indexados à plataforma PubMed. Para tanto, incluímos, aos descritores utilizados para a análise bibliométrica, os seguintes descritores: tactile or vibrotactile. Adicionalmente, utilizamos em nossa discussão 5 artigos indexados à plataforma Web of Science (MyRA), com a utilização de descritores iguais aos utilizados na plataforma PubMed.

A utilização das plataformas PubMed e Web of Science justifica-se pela natureza transdisciplinar da Tecnologia Assistiva, a qual envolve profissionais fisioterapeutas, terapeutas ocupacionais, fonoaudiólogos, professores, médicos, dentre outros (Sartoretto e Bersch, 2021). Assim, a utilização dessas plataformas permitiu uma busca ampla e a identificação de estudos que envolvessem diferentes profissionais que atuam no desenvolvimento e a utilização de recursos da Tecnologia Assistiva e o ensino de música para surdos.

Destacamos que a ATD propõe categorias que orientam a construção de textos analíticos, de forma organizada, e compreendem a unitarização e a categorização, que são as responsáveis por gerar os meta-textos analíticos (Moraes \& Galiazzi, 2006).

\section{Resultados}

A análise bibliométrica utilizada neste artigo permitiu verificar a produção ao longo do tempo sobre as pesquisas publicadas que relacionaram as áreas de música e surdez, bem como possibilitou mostrar quais as organizações, os autores que mais publicaram acerca da interseção dessas áreas temáticas e as palavras-chaves mais frequentes nessas pesquisas. O escopo dessa pesquisa compreendeu 721 artigos indexados à plataforma PubMed, com a utilização dos seguintes descritores: music; deaf or deafness. O Gráfico 1 mostra a produção científica de 1949 até 2021.

O Gráfico 1 mostra que o artigo indexado à plataforma PubMed mais antigo é de 1949. O ano de 2015 se destaca como o de maior número de publicações e o ano de 2007 se destaca como o ano de maior crescimento percentual em relação ao ano anterior $(230 \%)$.

Ressaltamos que esta pesquisa foi desenvolvida em maio de 2021 e podemos encontrar 15 artigos científicos já indexados. 
Gráfico 1 - Produção científica ao longo dos anos (1949 a 2021).

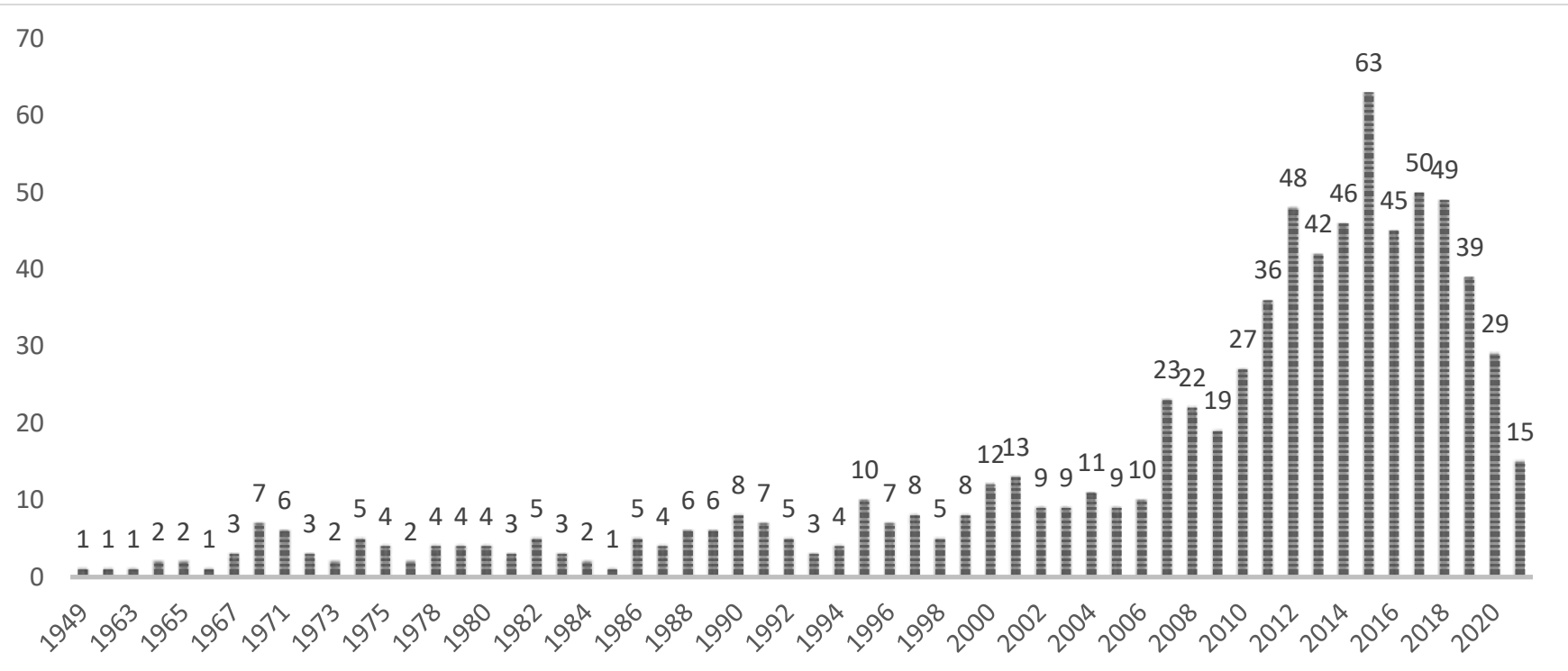

Fonte: Elaborado pelos autores a partir de dados da plataforma PubMed.

A análise de coautoria nos forneceu 578 organizações diferentes. As mais representativas se situam na China, nos Estados Unidos, na França e no Reino Unido, são elas: Shanghai Normal University, University of Reading, University Lyon 1, University of California e House Ear Institute of Los Angeles.

A Tabela 1 mostra a instituição, o país e o número de publicações das organizações mais representativas para o escopo desta pesquisa.

Tabela 1 - Instituições mais representativas em número de publicações.

$\begin{array}{lll}\text { Instituição } & \text { País } & \text { Número de publicações }\end{array}$

\begin{tabular}{lll}
\hline Shanghai Normal University & China & 5 \\
University of Reading & Reino Unido & 5 \\
University Lyon 1 & França & 4 \\
University of California & Estados Unidos da América & 3 \\
House Ear Institute of Los Angeles & Estados Unidos da América & 3 \\
\hline
\end{tabular}

Fonte: Elaborado pelos autores a partir de dados da plataforma PubMed.

A Tabela 1 mostrou que, dentre as organizações com 3 ou mais publicações dentro do escopo desta pesquisa, a Shanghai Normal University e a University of Reading são as que possuem o maior número, com 5 cada uma.

A University Lyon 1 aparece na sequência com 4 publicações e é seguida pela University of California e House Ear Institute of Los Angeles, com 3 cada uma. Destacamos que essas duas últimas são organizações que se situam no estado da Califórnia, nos Estados Unidos.

A análise de coautoria também nos forneceu dados sobre os autores que mais publicam sobre a interseção música e surdez. A Tabela 2 mostra os autores que possuem a autoria ou coautoria de 6 ou mais publicações. 
Tabela 2 - Autores que mais publicam sobre a interseção música e surdez.

$\begin{array}{ll}\text { Autor } & \text { Artigos }\end{array}$

Peretz, I.

Tillmann, B.

Caclin, A.

Paquette, S.

Gordon, K.

Liu, F.

Papsin, B.

\section{7}

10

8

7

6

6

6

Fonte: Elaborado pelos autores a partir de dados da plataforma PubMed.

A Tabela 2 apresentou os autores que possuem o maior o número de publicações dentro do escopo de nossa pesquisa, com destaque para Peretz, com 17 publicações.

A Figura 1, a seguir, mostra as redes de coautoria dos autores que mais publicam sobre a interseção música e surdez, com base nos dados fornecidos pela plataforma PubMed.

Figura 1 - Redes de coautoria dos autores que mais publicam sobre a interseção música e surdez.

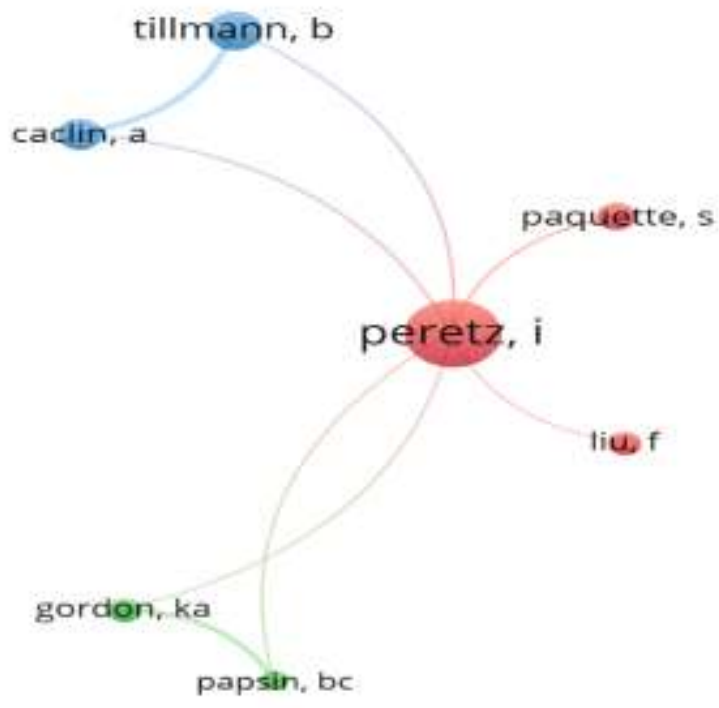

Fonte: VOSviewer, a partir de dados textuais da pesquisa no PubMed (2021).

Notou-se na Figura 1 que Peretz possui coautoria com os outros 6 autores que estão na lista de autores que mais publicam sobre a interseção música e surdez.

Destacamos que o software VOSviewer apontou 818 palavras-chaves para os 721 artigos científicos que obtivemos como resultado para os descritores: "music; deaf or defness".

A Figura 2 mostra o mapa de coocorrência com as principais palavras-chaves dos artigos publicados na plataforma PubMed que envolveram a interseção desses descritores. 
Figura 2 - Mapa de coocorrência de palavras-chaves dos dados da pesquisa na plataforma PubMed.

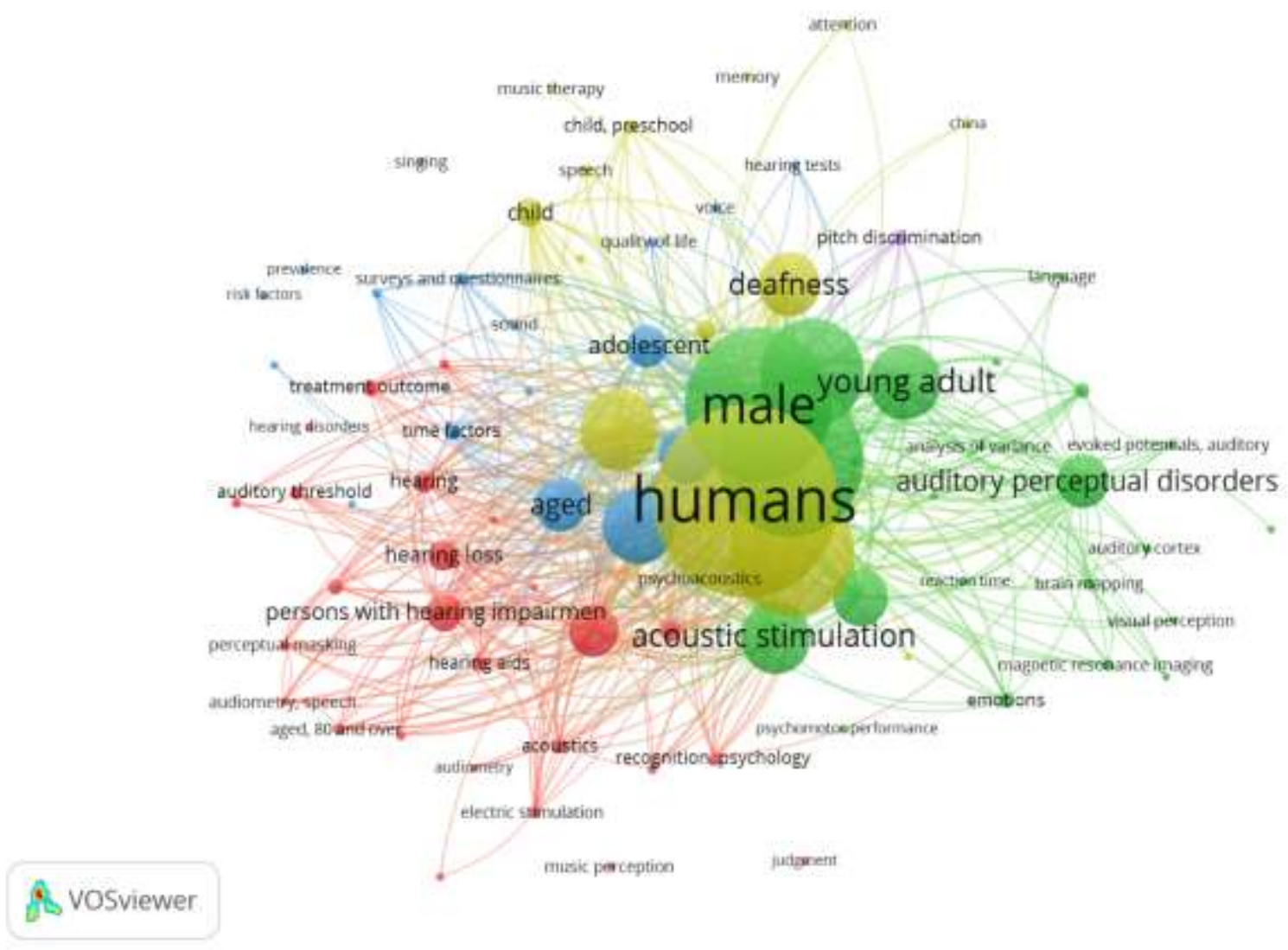

Fonte: VOSviewer, a partir de dados textuais da pesquisa no PubMed (2021).

Observa-se na Figura 2 que os artigos científicos que tratam a respeito da interseção música e surdez possuem, principalmente, palavras-chaves relacionadas à percepção de audição, de tom e de fala de seres humanos de ambos os sexos biológicos e de idades variadas, bem como referentes à utilização de implantes cocleares, estimulação elétrica e acústica.

A análise bibliométrica apontou dados quantitativos gerais sobre a interseção música e surdez. Nota-se a escassez de estudos sobre a utilização de Tecnologias Assistivas vibrotáteis na educação musical de surdos.

Desse modo, em atendimento ao objetivo geral deste artigo e a metodologia escolhida, optamos por inserir um descritor mais específico dentro de nosso escopo de pesquisa. Utilizamos tactile ou vibrotactile a fim de aprofundarmos a nossa discussão por meio da Análise Textual Discursiva.

Assim, encontramos 10 artigos científicos na plataforma PubMed, sendo que 7 deles possuem similitudes com o objeto de nossa pesquisa. Encontramos também 11 artigos científicos na plataforma Web of Science (MyRA), sendo que 5 novos artigos apresentaram relação com o nosso objeto. Diante disso, apresentaremos no Quadro 1 os doze artigos científicos que tratam sobre Tecnologias Assistivas vibrotáteis que podem ser utilizadas na educação musical de surdos.

Conforme mostra o Quadro 1, dentre os doze artigos científicos que tratam sobre Tecnologias Assistivas vibrotáteis que podem ser utilizadas na educação musical de surdos, nenhum dos autores participa de mais de uma publicação. Santos e Dantas (2017) salientam sobre a imprescindibilidade de divulgação e de descrição das funções das Tecnologias Assistivas para surdos, destacam a importância de haver melhor desenvolvimento da consciência geral acerca da inclusão e alertam sobre a necessidade de tanto surdos como ouvintes repensarem a utilização das Tecnologias Assistivas para essa finalidade, pois cada indivíduo é corresponsável pela inclusão. 
Quadro 1 - Artigos que tratam sobre Tecnologias Assistivas vibrotáteis que podem ser utilizadas na educação musical de surdos.

\begin{tabular}{|c|c|c|}
\hline Autores & Título do artigo científico & Plataforma \\
\hline Araujo, F. A. et al. (2017) & Auris System: Providing Vibrotactile Feedback for Hearing Impaired Population & Pubmed \\
\hline Ezawa M. (1988) & Rhythm perception equipment for skin vibratory stimulation & Pubmed \\
\hline $\begin{array}{l}\text { Good, A., Reed, M. J., \& Russo, F. } \\
\text { A. (2014) }\end{array}$ & Compensatory plasticity in the deaf brain: effects on perception of music & Pubmed \\
\hline $\begin{array}{c}\text { Karam, M., Russo, F. A., \& Fels, D. } \\
\text { I. (2009) }\end{array}$ & Designing the Model Human Cochlea: An Ambient Crossmodal Audio-Tactile Displa & Pubmed \\
\hline $\begin{array}{l}\text { Russo, F. A., Ammirante, P., \& } \\
\text { Fels, D. I. (2012) }\end{array}$ & Vibrotactile discrimination of musical timbre. Journal of experimental psychology & Pubmed \\
\hline $\begin{array}{l}\text { Sharp, A., Bacon, B. A., \& } \\
\text { Champoux, F. (2020) }\end{array}$ & Enhanced tactile identification of musical emotion in the deaf & Pubmed \\
\hline Tranchant, P. et al. (2017) & $\begin{array}{c}\text { Feeling the Beat: Bouncing Synchronization to Vibrotactile Music in Hearing and } \\
\text { Early Deaf People }\end{array}$ & Pubmed \\
\hline $\begin{array}{l}\text { Acton, Kelsie \& Howarth, Caroline } \\
\quad \& \text { Ouchi, Mieko. (2020) }\end{array}$ & Encountering Music Encountering Music: Songs Seen, Felt, and Heard & Web of Science \\
\hline Florian, H. et al. (2017) & Deaf people feeling music rhythm by using a sensing and actuating device & Web of Science \\
\hline Györgyjakab, M. (2018) & Music belongs to all of us! even to the the deaf! (?) & Web of Science \\
\hline Lucía, M. J. et al. (2020). & $\begin{array}{l}\text { Vibrotactile Captioning of Musical Effects in Audio-Visual Media as an Alternative } \\
\text { for Deaf and Hard of Hearing People: An EEG Study }\end{array}$ & Web of Science \\
\hline Petry, B. et al. (2018) & $\begin{array}{c}\text { Supporting Rhythm Activities of Deaf Children using Music-Sensory-Substitution } \\
\text { Systems }\end{array}$ & Web of Science \\
\hline
\end{tabular}

Fonte: Elaborado pelos autores a partir de dados das plataformas PubMed e Web of Science (MyRA).

Após fornecermos os dados quantitativos, os indicadores e as tendências de pesquisa, no próximo tópico, discutiremos a essência dos conteúdos dos artigos científicos apresentados no Quadro 1 com base na Análise Textual Discursiva.

\section{Discussão}

Após investigação da produção científica, discutiremos, por meio da Análise Textual Discursiva, as possibilidades e as limitações das Tecnologias Assistivas vibrotáteis para a educação musical de surdos, tendo como fundamento os doze artigos científicos apresentados no Quadro 1.

Para tanto, realizamos a categorização desses dados a fim de facilitar a compreensão e a interpretação. Nesse sentido, classificamos os conteúdos dos artigos sobre Tecnologias Assistivas vibrotáteis em três categorias, quais sejam: Tecnologias Assistivas vibrotáteis e sua relação com os aspectos fisiológicos; Tecnologias Assistivas vibrotáteis e sua relação com os fatores emocionais; Tecnologias Assistivas vibrotáteis e sua aplicação à educação musical. 
Em relação às Tecnologias Assistivas vibrotáteis e sua relação com os aspectos fisiológicos, conforme aponta Györgyjakab (2018), os surdos não podem ouvir a música, mas podem vê-la e senti-la se lhes fornecermos os elementos visuais e vibrotáteis corretos. Isso é corroborado por Acton, Howarth \& Ouchi (2020), que salientam que a música e o som não se restringem a elementos auditivos, mas também a aspectos vibrotáteis e visuais. Russo, Ammirante \& Fels (2012) corroboraram esses dados ao investigaram a habilidade de discriminar timbres musicais com base apenas na estimulação vibrotátil.

Segundo Good, Reed \& Russo (2014), o aprimoramento específico das habilidades visuais e vibrotáteis em indivíduos surdos pode fornecer processamento diferencial de aspectos não auditivos da performance musical. Esses autores reforçam as evidências de haver plasticidade compensatória em indivíduos surdos e asseveram que os estímulos visuais e vibrotáteis podem ser processados nos centros auditivos do cérebro. Sharp, Bacon, \& Champoux (2020) corroboram o fato de que a plasticidade cerebral após a surdez pode levar a uma habilidade tátil complexa melhorada.

Os estudos aqui analisados buscaram extrair informações musicais do áudio e criar uma representação musical a partir de diferentes estímulos vibrotáteis para fornecer experimentação musical para pessoas que possuem algum tipo de perda auditiva. Um exemplo disso é o sistema Auris, apresentado por Araujo, F. A. et al. (2017), que, por meio de uma metodologia de teste baseada em um registro não invasivo da atividade cerebral usando um dispositivo eletroencefalográfico, se propõe a apresentar um novo formato de mídia musical a ser interpretado por outros sentidos que não a audição.

A respeito dessas Tecnologias Assistivas, Ezawa (1988) descreve, além das características da estimulação vibrotátil, um dispositivo experimental para a transmissão de informações rítmicas contidas na música por meio da estimulação vibrotátil. Nessa esteira, Petry et al. (2018) apontam que os sistemas vibrotáteis se diferenciam principalmente pela localização no corpo onde o usuário percebe o estímulo vibrotátil.

Karam, Russo \& Fels (2009) apresentaram um modelo de Tecnologia Assistiva vibrotátil para apoiar a tradução de músicas para surdos. Esse modelo foi usado para traduzir música em sinais de vibração discretos exibidos ao longo da parte de trás do corpo e utiliza também um monitor tátil ambiente. Os autores concluíram que a reação de surdos à Tecnologia Assistiva vibrotátil proposta foi positiva e que obtiveram comentários e sugestões da comunidade de surdos em relação às diferentes aplicações e maneiras de tornar o dispositivo mais confortável e acessível universalmente.

Quanto às Tecnologias Assistivas vibrotáteis e sua relação com os fatores emocionais, Sharp, Bacon \& Champoux (2020) se propuseram a investigar a identificação tátil da emoção musical em surdos. Para tanto, compararam participantes surdos e ouvintes com privação da audição quanto à identificação de três das quatro emoções testadas: tristeza; medo; tranquilidade; felicidade. Os participantes avaliaram as melodias com base em sua percepção emocional por meio de estímulos apresentados por meio de uma luva tátil. Os resultados sugeriram uma identificação tátil aprimorada da emoção musical em surdos.

Segundo Lucía et al. (2020), as mesmas áreas cerebrais, embora em hemisférios diferentes, são ativadas em participantes ouvintes e em participantes surdos ao assistir ao mesmo vídeo com trilha sonora e com uma estimulação vibrotátil suave e rítmica na palma da mão e nas pontas dos dedos. Os autores concluíram que a Tecnologia Assistiva vibrotátil como um possível canal para transmitir as informações emocionais contidas em uma trilha sonora audiovisual e, com efeito, provocar uma maior reação emocional em surdos.

A respeito das Tecnologias Assistivas vibrotáteis e sua aplicação à educação musical de surdos, dos artigos encontrados e analisados a respeito desse tema, foi Ezawa (1988) quem primeiro discorreu sobre a aplicação de estímulos vibrotáteis para estudantes de música surdos. Por meio de testes clínicos, foram relatados resultados da aplicação desses estímulos em estudantes de escolas para surdos para verificar se as informações rítmicas contidas na música eram semelhantes às proporcionadas pelos dispositivos vibrotáteis.

Petry et al. (2018) destacam que as Tecnologias Assistivas vibrotáteis captam a música e a mapeiam para um ou mais canais sensoriais alternativos, preservando uma ou mais características-chave da música, por exemplo, o ritmo. Esse estudo 
apontou que os estudantes surdos ficaram mais confiantes e mais engajados nas aulas de música com a utilização da Tecnologia Assistiva vibrotátil que propuseram. Essa proposta foi a de um dispositivo de substituição sensorial-musical visual e vibrotátil, que promove a discriminação de ritmo, reprodução e expressividade de surdos.

A esse respeito, Araujo et al. (2017) asseveram que dispositivos vibrotáteis podem facilitar a compreensão sobre a cognição musical humana a fim de melhorar a precisão de representação musical. Segundo Petry et al. (2018), o ritmo é o primeiro conceito musical que os surdos aprendem nas aulas de música. Esses autores destacam que a surdez pode criar uma lacuna no ciclo de feedback, pois limita as informações auditivas que os músicos surdos podem empregar para avaliar seu desempenho.

Florian, H. et al. (2017) apresentaram uma Tecnologia Assistiva para ajudar os surdos a sentir o ritmo da música. A Tecnologia Assistiva inclui um sensor de áudio, dois microcontroladores, com saídas de luz e vibrotátil, para transmitir a sensação do ritmo da música.

Para a educação musical, é importante a evidência trazida por Good, Reed \& Russo (2014) de que os elementos não auditivos da música podem fornecer uma experiência, para os surdos, que é qualitativamente comparável às experiências que uma pessoa ouvinte tem enquanto ouve música.

Good, Reed \& Russo (2014) salientam que a utilização de modalidades visuais e vibrotáteis pode ser ainda mais aprimorada por meio do uso de Tecnologia Assistiva. Esses autores ressaltaram que habilidades sensoriais únicas proporcionadas pelo cérebro do surdo devem ser adotadas ao desenvolver novas músicas destinadas a incluir a comunidade surda, com o propósito de promover a arte da música para todos.

No entanto, Acton, Howarth \& Ouchi (2020) alertam que, ainda que haja convergência dos sentidos sobre as concepções de som e música, há ainda muita desigualdade em relação ao acesso de surdos a eventos predominantemente musicais.

Tranchant, P. et al. (2017) investigaram a sincronização de batidas com a música eletrônica vibrotátil em surdos e ouvintes por meio da dança. Esses autores testaram sete surdos e quatorze ouvintes quanto à capacidade de pular no tempo dos estímulos vibrotáteis, sem som, entregues por meio de uma plataforma vibratória. Adicionalmente, os estímulos auditivos correspondentes, sem vibrações, foram usados em uma condição adicional no grupo de ouvintes. Os resultados mostraram que a sincronização tátil-motora, na dança, ocorre independentemente da experiência auditiva.

Conforme assevera Otero Caicedo (2021), os surdos nos fornecem outras compreensões da música e nos convidam a vivenciá-la de lugares não registrados.

Nesse sentido, a oferta de educação musical aos surdos não somente é uma questão de inclusão educacional e social, mas também é uma possibilidade de proporcionar, aos ouvintes, vivenciar a música de outras formas, por meio de outros sentidos.

\section{Considerações Finais}

Neste artigo, pretendemos investigar as possibilidades e as limitações das Tecnologias Assistivas vibrotáteis para a educação musical de surdos, por meio de análise bibliométrica e de análise textual descritiva.

Com base em nossa pesquisa quantitativa, por meio da bibliometria, concluímos que há uma grande quantidade de dados disponíveis sobre a interseção música e surdez capaz de fornecer informações aos pesquisadores da área de educação musical de surdos e aos que constituem a comunidade interessada: os próprios surdos. Nossa pesquisa constatou não apenas dados, mas também tendências e indicadores de pesquisa científica que trataram de um ponto em comum: a interseção música e surdez.

Notamos que a literatura científica sobre essa interseção se constitui de um corpus relevante, com dados de extrema importância. Observamos uma tendência crescente que indica alta do número de artigos científicos publicados nessa área. Destacamos que essa tendência pode indicar tanto o aumento real da produção na área como pode indicar apenas o aumento do 
número de periódicos indexados à plataforma PubMed.

A partir da Análise Textual Discursiva, classificamos os conteúdos dos artigos sobre Tecnologias Assistivas vibrotáteis em três categorias: Tecnologias Assistivas vibrotáteis e sua relação com os aspectos fisiológicos; Tecnologias Assistivas vibrotáteis e sua relação com os fatores emocionais; Tecnologias Assistivas vibrotáteis e sua aplicação à educação musical.

Quanto a análise categorial, nosso posicionamento é o de que o fato de as Tecnologias Assistivas vibrotáteis poderem ser processados nos centros auditivos do cérebro, por meio da plasticidade neural, fundamenta a interpretação de que esses dispositivos podem contribuir para a educação musical de surdos. Sugerimos pesquisas empíricas longitudinais que avaliem a efetividade da utilização dessas Tecnologias Assistivas no contexto escolar.

\section{Referências}

Acton, K. \& Howarth, C. \& Ouchi, M. (2020). Encountering Music Encountering Music: Songs Seen, Felt, and Heard. Canadian Theatre Review. 184. 15. $10.3138 /$ ctr.184.003.

Ângulo-Cuentas, G. L.; Galvis-Lista, E. A.; González- Zabala, M. P. \& Fuentes-Cuadrado, C. V. (2018). Análisis bibiliométrico: salud y calidad de vida. 1. ed. Universidade del Magdalena, Santa Marta, Colombia.

Araujo, F. A., Brasil, F. L., Santos, A. C. L., Batista Junior, L. S., Dutra, S. P. F., \& Batista, C. E. C. F. (2017). Auris System: Providing Vibrotactile Feedback for Hearing Impaired Population. BioMed research international, 2017, 2181380. https://doi.org/10.1155/2017/2181380.

Borowiec, J. \& Hökelmann, A. \& Osiński, W. (2019). The Level of Self-Esteem of Deaf Children: Can Participating in Dance Lessons with Vibrational Headphones Improve It?. The Arts in Psychotherapy. 64. 10.1016/j.aip.2019.03.004.

Brasil. (2015). Lei no 13.146, de 6 de jul. de 2015. Lei Brasileira de Inclusão da Pessoa com Deficiência. Diário Oficial da União, 7 de julho de 2015. Disponível em: http://www.planalto.gov.br/ccivil_03/_Ato2015-2018/2015/Lei/L13146.htm; acesso em: 23 de junho de 2021.

Ezawa M. (1988). Rhythm perception equipment for skin vibratory stimulation. IEEE engineering in medicine and biology magazine: the quarterly magazine of the Engineering in Medicine \& Biology Society, 7(3), 30-34. https://doi.org/10.1109/51.7932.

Florian, H.; Mocanu, A.; Vlasin, C.; Machado, J.; Carvalho, V.; Soares, F.; Astilean, A. \& Avram, C. (2017). Deaf people feeling music rhythm by using a sensing and actuating device. Sensors and Actuators A: Physical, v. 267. https://doi.org/10.1016/j.sna.2017.10.034.

Good, A., Reed, M. J., \& Russo, F. A. (2014). Compensatory plasticity in the deaf brain: effects on perception of music. Brain sciences, 4(4), 560-574. https://doi.org/10.3390/brainsci4040560.

Gesser, A. (2009). LIBRAS? que língua é essa? Crenças e preconceitos em torno da língua de sinais e da realidade surda. São Paulo: Parábola.

Goffman, E. (2008). Estigma: notas sobre a manipulação da identidade deteriorada. Trad. Márcia Bandeira de Mello Leite Nunes. 4.ed. Rio de Janeiro: LTC.

Györgyjakab, M. (2018). Music belongs to all of us! even to the the deaf! (?). Studia Universitatis Babes-Bolyai - Musica, n. 2. https://www.ceeol.com/search/article-detail?id=722854.

Karam, M., Russo, F. A., \& Fels, D. I. (2009). Designing the Model Human Cochlea: An Ambient Crossmodal Audio-Tactile Display. IEEE transactions on haptics, 2(3), 160-169. https://doi.org/10.1109/TOH.2009.32

Lucía, M. J. et al. (2020). Vibrotactile Captioning of Musical Effects in Audio-Visual Media as an Alternative for Deaf and Hard of Hearing People: An EEG Study. IEEE Access, vol. 8. doi: 10.1109/ACCESS.2020.3032229.

Martias, Z. (2017). The Contribution of Playing Music Notation toward the Development of Sound Perception on Students with Hearing Impaired. Advances in Social Science, Education and Humanities Research (ASSEHR), volume 148. https://doi.org/10.2991/icla-17.2018.63. Disponível em: https://www.atlantispress.com/article/25888959.pdf.

Moraes, R. \& Galiazzi, M. C. (2006). Análise textual discursiva: processo reconstrutivo de múltiplas faces. Ciência \& Educação (Bauru), 12(1),117-128. Disponível em: https://doi.org/10.1590/S1516-73132006000100009.

Otero Caicedo, L. E. (2021). La música que des-cubre el silencio: Pedagogías decoloniales para la educación musical de personas sordas. Calle 14 revista de investigación en el campo del arte, 16(29), 118-127. https://doi.org/10.14483/21450706.17407.

Paula, T. R. M. de; Pederiva, P. L. M. (2018). Sou surdo e gosto de música: a musicalidade da pessoa surda na perspectiva histórico-cultural. Editora Appris. Edição do Kindle.

Petry, B.; Illandara, T.; Elvitigala, D. S. \& Nanayakkara, S. (2018). Supporting Rhythm Activities of Deaf Children using Music-Sensory-Substitution Systems. In Proceedings of the 2018 CHI Conference on Human Factors in Computing Systems (CHI '18). Association for Computing Machinery, New York, NY, USA, Paper 486, 1-10. DOI:https://doi.org/10.1145/3173574.3174060.

Ribeiro, H. C. M. (2017). Bibliometria: quinze anos de análise da produção acadêmica em periódicos brasileiros. Biblios, 0(69), 1-20. https://dx.doi.org/10.5195/biblios.2017.393. 
Research, Society and Development, v. 10, n. 8, e51710816765, 2021

(CC BY 4.0) | ISSN 2525-3409 | DOI: http://dx.doi.org/10.33448/rsd-v10i8.16765

Russo, F. A., Ammirante, P., \& Fels, D. I. (2012). Vibrotactile discrimination of musical timbre. Journal of experimental psychology. Human perception and performance, 38(4), 822-826. https://doi.org/10.1037/a0029046.

Santos, P. K. \& Dantas, N. M. R. (2017). Tecnologias assistivas e a inclusão do estudante surdo na educação superior. Revista Internacional de Educação Superior, 3(3), 494-514. DOI: https://doi.org/10.22348/riesup.v3i3.7793.

Sartoretto, M. L. \& Bersch, R. (2021). Assistiva: tecnologia e educação. Disponível em: https://www.assistiva.com.br/tassistiva.html. Acesso em 23 de junho de 2021.

Sharp, A., Bacon, B. A., \& Champoux, F. (2020). Enhanced tactile identification of musical emotion in the deaf. Experimental brain research, 238(5), 12291236. https://doi.org/10.1007/s00221-020-05789-9.

Silva, N. M. da, Alves, J. F., Castro, A. B. C. de, \& Varela, J. H. S. (2020). Educação musical de surdos: características, barreiras e práticas exitosas. Educação e Pesquisa, 46. Disponível em: https://doi.org/10.1590/S1678-4634202046221995.

Tranchant, P., Shiell, M. M., Giordano, M., Nadeau, A., Peretz, I., \& Zatorre, R. J. (2017). Feeling the Beat: Bouncing Synchronization to Vibrotactile Music in Hearing and Early Deaf People. Frontiers in neuroscience, 11, 507. https://doi.org/10.3389/fnins.2017.00507.

Vigotski , L. S. (2001). A defectologia e o estudo do desenvolvimento e da educação da criança anormal. Educação e Pesquisa, São Paulo, v. 37, n. 4, p. 861870. https://www.scielo.br/j/ep/a/x987G8H9nDCcvTYQWfsn4kN/?lang=pt. 